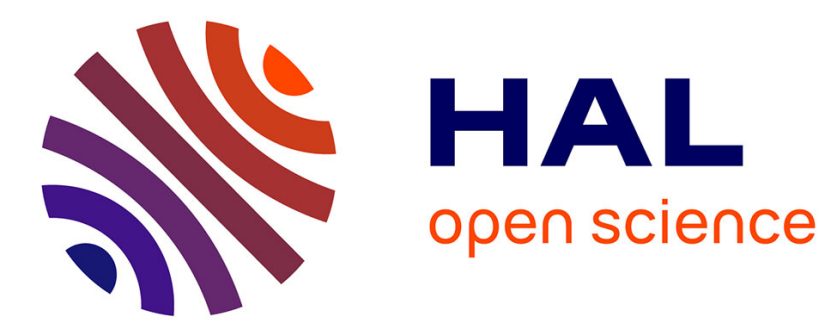

\title{
On the design and optimization of acoustic network resonators for tire/road noise reduction
}

Bin Wang, Denis Duhamel

\section{To cite this version:}

Bin Wang, Denis Duhamel. On the design and optimization of acoustic network resonators for tire/road noise reduction. Applied Acoustics, 2017, 120, pp.75 - 84. 10.1016/j.apacoust.2017.01.017 . hal-01695358

\section{HAL Id: hal-01695358 \\ https://hal.science/hal-01695358}

Submitted on 29 Jan 2018

HAL is a multi-disciplinary open access archive for the deposit and dissemination of scientific research documents, whether they are published or not. The documents may come from teaching and research institutions in France or abroad, or from public or private research centers.
L'archive ouverte pluridisciplinaire HAL, est destinée au dépôt et à la diffusion de documents scientifiques de niveau recherche, publiés ou non, émanant des établissements d'enseignement et de recherche français ou étrangers, des laboratoires publics ou privés. 


\title{
On the design and optimization of acoustic network resonators for tire/road noise reduction
}

\author{
Bin Wang ${ }^{\mathrm{a}}$, Denis Duhamel ${ }^{\mathrm{a}, *}$ \\ ${ }^{a}$ Université Paris-Est, Laboratoire Navier, ENPC-IFSTTAR-CNRS, UMR 8205, Ecole des \\ Ponts ParisTech, 6-8 avenue Blaise Pascal, 77455 Marne-la-Vallée, France
}

\begin{abstract}
In this work we propose a numerical method for the calculation of resonant frequencies of network resonators and we also present an optimization method based on genetic algorithms to get targeted resonant frequencies of the network resonators. We can optimize parameters of the network structure such as junction types and end positions. Experiments are conducted on optimized wooden network resonators to validate the method. Good agreement is found between the measured and targeted resonant frequencies. Applications to tire/road noise are considered.

Keywords: Acoustic network resonator, End correction, Resonant frequency, Optimization
\end{abstract}

\section{Introduction}

Tire treads and road textures in the contact zones between tires and roads can be considered as acoustic network resonators. Consequently, the acoustic fields around the tire/road systems are influenced by network resonances. These network resonances in the contact zone in Fig 1 are seen as one of the noise enhancement mechanisms in [1]. Since the network resonators in the contact zone have large influence on the acoustic fields around their resonant frequencies, the network resonators should be investigated in detail. First studies of some

\footnotetext{
* Corresponding author

Email address: denis.duhamel@enpc.fr (Denis Duhamel)
}

Preprint submitted to Applied Acoustics

October 14, 2016 
simple acoustic resonators are reviewed as follows.

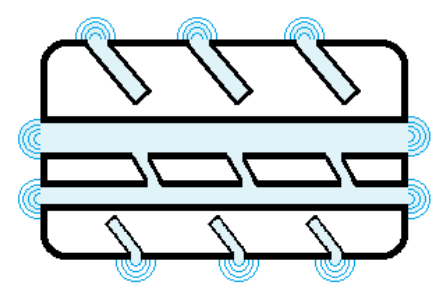

Figure 1: Network resonators in the contact zone between a tire and a road

Besides porous materials and perforated panels, narrow quarter-wave tube resonators are also widely used for the sound absorption in a wall or panel for a narrow frequency band based on the resonance of air inside the tube and the viscous shear and thermal conductivity losses on the tube walls. The model by Zwikker and Kosten 2 for wave propagation in cylindrical tubes included 15 the viscosity and thermal conductivity. Tijdeman [3] proved that this model is complete and accurate for both narrow and wide tubes. Eerden [4] studied the influence of the viscous and thermal conductivity losses on the absorption coefficient and concluded that the viscothermal effects cannot be neglected if the resonators are used for sound absorption because they result in energy being dissipated and the effective speed of sound inside the tube can be considerably reduced. Around the resonant frequencies, we can see a maximum sound absorption. The theory and applications of quarter-wave resonators are summarized in [5].

The quarter-wave tube has an open and a closed end, but resonators with two open ends can also be used for the sound absorption, especially for the case where air needs to be transported through walls or one needs to see through the wall. Eerden [4] studied this case, and concluded that at low frequencies $(f<2000 \mathrm{~Hz})$ the waves propagating in the resonator are not absorbed at the end but are reflected back into the resonator due to the mass reactance at so the free end. For higher frequencies $(2000-10000 \mathrm{~Hz})$ the waves are absorbed due to radiation into infinity. In order to create broadband sound absorption, coupled tube resonators with different cross-sectional areas and lengths can be 
applied. The mechanism for the broadband absorption is that the sound energy is dissipated by the viscothermal effects and the incident waves are cancelled due to the broadband resonance of air in the coupled resonators.

Helmholtz resonators (HRs) are also used to control the noise inside enclosures in many studies. Helmholtz resonators can be considered as a mass-spring system. The spring stiffness is represented by the volume of air and the mass is given by the small column of vibrating air in a perforation of the panel. The energy can be dissipated by the vibrating air and the porous material placed in the volume. See [6, 7, 8, 9, 10, for different applications of these Helmholtz resonators to noise reduction. More specifically T-shaped acoustic resonators can be seen in many studies for noise control in small enclosures, see for instance [11, 12, 13, 14, 15, 16] for models and experimental results on these resonators.

If we are interested more specifically in tire noise, today the noise due to the vibrations of a rolling tire can be calculated with convincing accuracy. However, air pumping is not understood very well. In [17 Hayden described the air movement in the contact zone between a rolling tire and a road. Air is squeezed out when the treads at the entrance of the contact zone are compressed on the road surface, and flows into the voids when the treads lift up from the road surface. Daffayet et al. [18 measured the pressure in cylindrical cavities over which a smooth tire rolled. They assumed that the noise is generated by opening and closing the cavities in the contact zone. Ronneberger [19] thought that air was displaced by the changing gaps between the tire and road surfaces, because the treads are deformed by road roughness. These sources are located in the contact zone between the tire and the road and the sound is modified by the horn effect.

Horn effect is an essential noise enhancement mechanism. The tire/road system can be seen as a horn-like structure. The surfaces of the tire and the road constitute horns in front of and behind the contact zone. The noise generated in the contact zone is amplified by the multiple reflections between the tire surface and the road surface which are acoustically reflecting surfaces. The amplification of the horn effect reaches up to 10 to $20 \mathrm{~dB}$ in the results of previous studies, 
where the road and the tire are modeled with smooth surfaces. A first attempt at an analytical description of the horn effect was made by Ronneberger [20]. Kropp et al. 21] suggested a theoretical model based on multipole synthesis. The model can provide a reasonable prediction of noise levels at mid and high frequencies for a tire placed on a hard surface. However, it overestimates the horn amplification effect at low frequencies. Graf et al. [22, 23] first investigated experimentally the horn amplification of sound generated by a simple acoustic source. The boundary element method is then shown to give predictions. The dependence of the horn-effect on different geometrical parameters is also investigated both through experiments and boundary element calculations. It shows that for the intermediate frequency range the BEM provides an excellent tool 75 to calculate the horn effect for practical geometries. The aim of the work by Anfosso et al. [24, 25] is also to predict the amplification due to horn effect. Sound pressure amplification of a 2D infinite rigid cylinder is obtained using the analytical approach based on modal decomposition of sound pressure. It gives quick and accurate results, but is limited to simple geometrical configurations and purely reflecting properties of boundaries. In [26] Fadavi et al deal with the horn effect using a 3D cylinder tire model. The sound pressure and sound amplification are calculated in the space around the 3D tire model using the Boundary Element Method. The influence of different parameters such as the position and size of the source are studied in terms of amplification and sound pressure spectrums. All these studies are made for smooth roads and tires and do not take into account the real geometry of the tire or the road.

In this work, we want to estimate the influence of non smooth geometries on the horn effet. For this, we focus on network resonators and use several assumptions for the networks. There are only right-angled junctions in the networks. The pipes in the networks have the same cross-section. The networks could have open or closed ends. For the open ends, end corrections depend on flange shapes. So, first, methods for the calculation of end corrections will be introduced in section 2 Next, in section 3, a numerical method for the calculation of resonant frequencies of network resonators will be developed. Then, 
in section 4 an optimization method will be proposed to get the targeted resonant frequencies. Some examples of the application of the optimization method are shown in section 5 while comparisons with experimental measurements are given in section 6. Last some conclusions will be given.

\section{Determination of end corrections}

100 cies of a network with open ends. A short distance should be added to each end of the network to get precise results. This short distance is called the end correction, which makes each straight part of the network a little longer than its physical length.

resonances. The sound waves are reflecting at open ends, which are not perfectly at the end sections of the network, but at small distances (end corrections) outside the network.

The end corrections of the network open ends can be obtained from the radiation impedances which have small but finite values by (1) from 27.

$$
\tilde{\delta}=\boldsymbol{R e}\left[k^{-1} \arctan \left(\frac{-Z_{r}}{i \rho c}\right)\right]
$$

The upper script $\sim$ means that it is a frequency-dependent quantity. Here only the present study. To estimate the end corrections of the network, the radiation impedances of the open ends should be calculated first by the impedance transfer equation of an acoustic transmission line (2) from [27, because $Z_{r}$ cannot be calculated or measured directly at the pipe end.

$$
Z_{r}=-i \rho c \tan \left[\arctan \left(\frac{-Z_{l}}{i \rho c}\right)-k l\right]
$$

$Z_{l}$ is the impedance at an abscissa $x=-l$, i.e., at a distance $l$ from the open end. It can be calculated as Dalmont did using a BEM numerical method [27.

Equation (2) means that the radiation impedance can be obtained from the case where $Z_{l}$ is an input impedance of a pipe of length $l$. Then the end 
corrections can be obtained by (1). The values of end corrections depend on flange shapes. An open pipe end with different flange shapes has different end corrections. In this section, end corrections of a network with complex flanges are calculated by Dalmont's methods or by the BEM. The flanges are a round surface and a plane surface (see Fig 2), which will be used in section 4 The network is shown in red. Some pipes are identified by the numbers one to three shown in green.

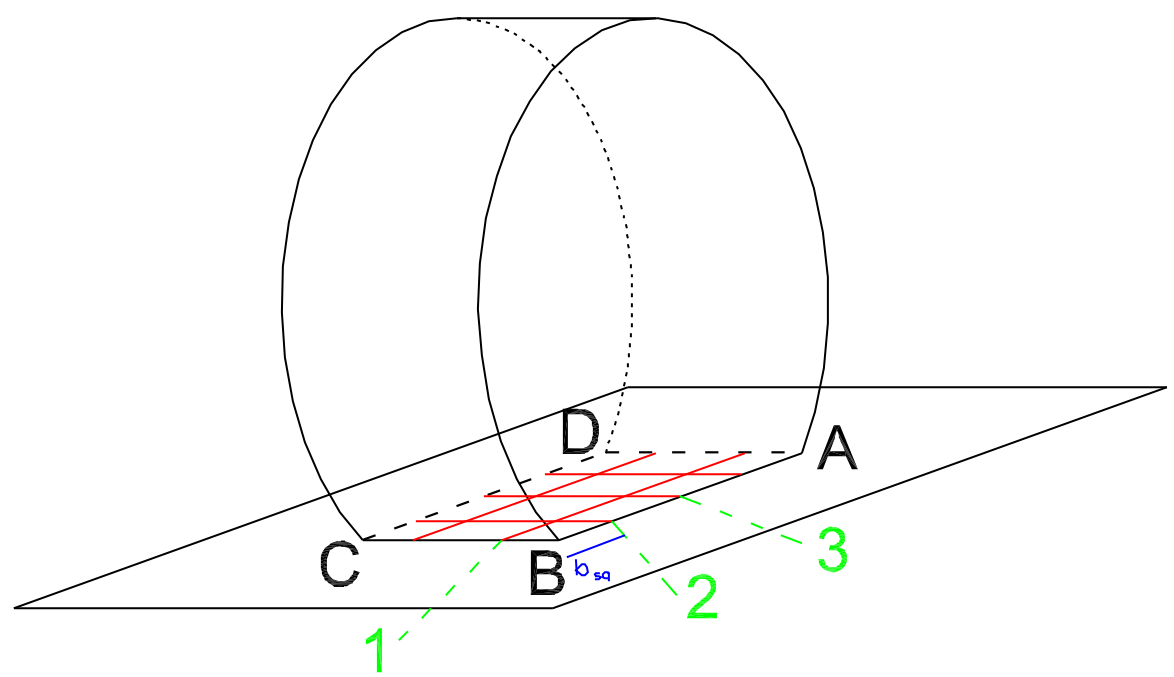

Figure 2: A network (in red) between a round surface and a rigid plane surface

Only the end corrections of longitudinal pipes will be discussed. For transverse pipes they can be calculated approximately by Dalmont's fit formula (3) for rectangular flanges because the flange of transverse ends is flat.

$$
\delta_{s q}=\delta_{s q \infty}+\frac{a_{s q}}{b_{s q}}\left(\delta_{s q 0}-\delta_{s q \infty}\right)+0.057 \frac{a_{s q}}{b_{s q}}\left[1-\left(\frac{a_{s q}}{b_{s q}}\right)^{5}\right] a_{e f f}
$$

with $\delta_{s q \infty}=0.811 a_{e f f}$ and $\delta_{s q 0}=0.597 a_{e f f}$, in which $a_{e f f}=2 a_{s q} / \sqrt{\pi}$. Here, $2 b_{s q}$ is the flange width, respectively. $b_{s q}$ is the shorter one of the two distances 
between the transverse end and the sides $A D$ or $B C$ in Fig 2 for which the value of $b_{s q}$ for pipe number 2 is shown. $2 a_{s q}=0.009 \mathrm{~m}$ is the pipe width which is small and not shown in the figure.

The longitudinal pipes at different positions have different shapes and dimensions of the flanges. Although their ends may not be in the middle of the flanges, in the interest of simplicity they are approximately assumed in the middle of the flanges. The round surface in Fig 2 is a cylinder. In the contact zone, the cylinder surface is flat.

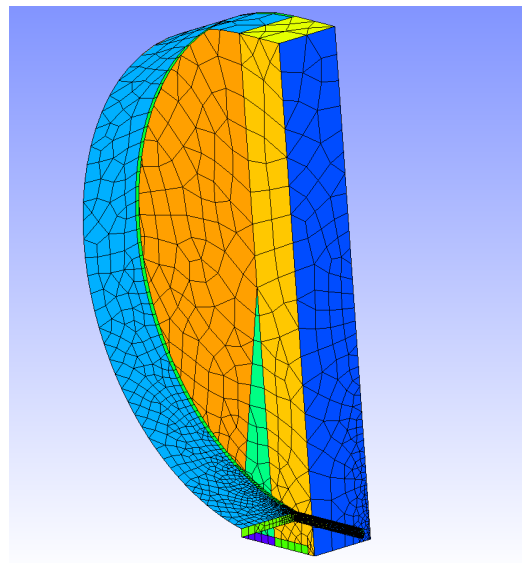

(a)

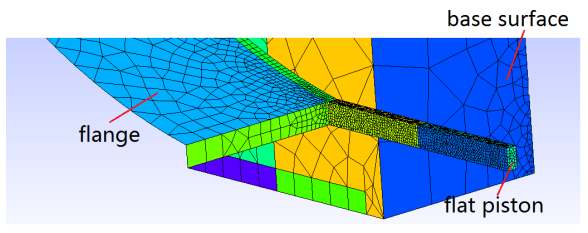

(b)

Figure 3: (a) Half a mesh of the boundary element model of a rectangular tube with a cylindrical flange; (b) Details of the mesh of the tube.

In order to calculate the end corrections of longitudinal pipes, half a cylinder is meshed for BEM, which is shown in Fig $3 \mathrm{a}$ and $3 \mathrm{~b}$. This is a longitudinal pipe, like pipe 1 in Fig 2, but located at the center of the contact zone. Since the plane surface is rigid, it can be considered as a symmetric plane. A symmetric plane of the cylinder exists, so only half of the cylinder is meshed. The radius of the cylinder is $0.27 \mathrm{~m}$. The half pipe has rectangular cross-section with area of $0.0045 \mathrm{~m} \times 0.009 \mathrm{~m}$. The length is $0.1 \mathrm{~m}$. It has an open end and a closed end which has a unit velocity as boundary condition. The base surface behind the piston is modelled as flat to reduce the elements because the flange shape 

pipe. It has a very small impedance which is used to minimize the reflections. Other parts of the model are rigid.

The acoustic pressure at the central node of the closed end is calculated by a BEM software. This software was developped by the authors and some details on the formulation which has been used can be found in 28. For the computations in this article, first order elements of triangular or quadrangular shapes were used. The number of nodes was approximatly 1600 and the number of elements 2900. This was found sufficient for the frequency band which is studied in the following.

The acoustic velocity at the closed end of the pipe is unity, so the impedance of the piston is obtained. Then the radiation impedance is calculated from this impedance by equation 2 .

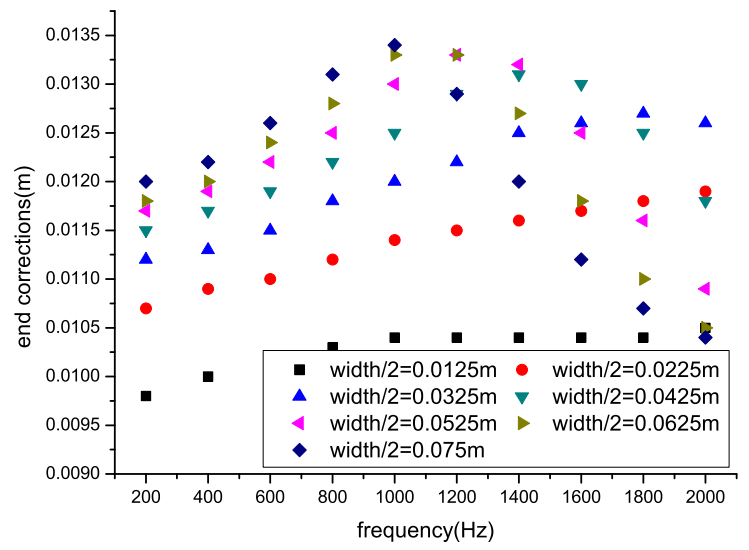

Figure 4: End corrections of longitudinal pipes with cylindrical flanges of different widths within $2000 \mathrm{~Hz}(\mathrm{ka}<0.23)$.

The end corrections of the longitudinal pipe for cylinders of different widths are shown in Fig 4 for $k a<0.23$, and the frequency interval is $200 \mathrm{~Hz}$. They are obtained by BEM as no analytical formula can be used in this case. For each width of the cylinder, the end corrections are frequency-dependent, but their standard deviations are very small (see Fig 5), which indicates that they tend to be very close to the mean values. The standard deviation is calculated by 


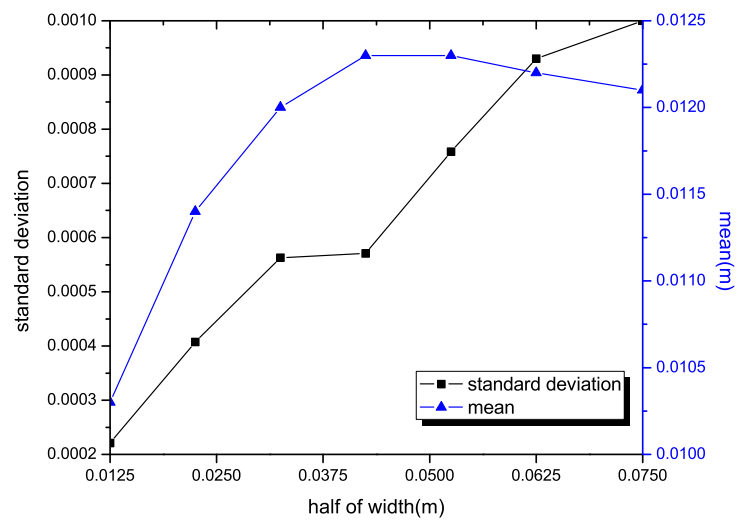

Figure 5: Standard deviations and mean values of end corrections of longitudinal pipes with cylindrical flanges of different widths.

equation (4). So the mean values of end corrections for each width in Fig 5 are used to get a fit formula of end corrections (5), in which $w$ is half of the width.

$$
\sigma=\sqrt{\frac{1}{N} \sum_{i=1}^{N}\left(x_{i}-\mu\right)^{2}}
$$

with $\mu=\frac{1}{N} \sum_{i=1}^{N} x_{i}$, where $x_{i}$ is the end correction at each frequency and $N$ is the number of frequencies calculated.

$$
\delta=0.00808+0.22128 w-3.72112 w^{2}+19.80897 w^{3}
$$

This fit formula will be used in section 4. The same procedure can be used to get fit formulas for other pipes and flanges of different shapes and dimensions.

\section{Calculation of resonant frequencies}

In the network, there are columns of air. Waves traveling in air will reflect back when they reach the end. A standing wave is created at the resonant frequency of the network. An open network will resonate if there is an antinode at each open end. These anti-nodes are places where there are maximum velocities and minimum pressures $(p=0)$. However, these zero pressures are not at the physical ends of the network as they are altered by their contacts with air. In fact they are at the end correction positions of the network. 
Knowing the resonant frequencies of the network is essential. The network has a great influence on the exterior acoustic field around the resonant frequencies, and at other frequencies there is no change of acoustic pressure. In order to calculate this influence the resonant frequencies should be estimated first, otherwise all the frequencies should be calculated and it leads to large computations.

There are analytical solutions for simple pipes such as a straight pipe, a T-shaped pipe and so on. However, numerical methods should be adopted in order to get the resonant frequencies of complex networks. It is assumed that there is a plane wave in the straight parts of the network for the cross-sections are quite small compared to the wavelengths.

Merkli [1] proposed a simple model to calculate the resonant frequencies of a T-shaped resonator by using the wave propagation theory. In this model, end corrections for open ends should be considered. Besides, the end correction of each branch at the junction position should also be included in the model. So for each open branch, the effective length includes the physical length of the branch, the end correction at the junction position and the end correction of the open end. Dalmont's [27] methods and results can be used for the calculations of the open end corrections. With his methods, the open end flanges could have any shape and dimension. The end corrections at the junction position can be calculated with the model of $\mathrm{Li}[12$.

Theoretically, Merkli's model can be applied to other cases such as a Lshaped junction pipe, a cross junction pipe or a network. However before estimating the resonant frequencies, the end corrections of branches at the junction positions should be calculated precisely. Existing models can only give approximate estimations of these interior end corrections. If we use Merkli's model to calculate the resonant frequencies of a network, there will be large errors due to the approximate interior end corrections. In order to avoid using these interior corrections, numerical methods should be adopted for the calculation of the resonant frequencies.

The resonant frequencies can be obtained by solving an eigenvalue problem 
(6) of an undamped finite element model.

$$
\left(K-\omega^{2} M\right) \phi=0
$$

$K$ is the stiffness matrix, $M$ is the mass matrix, $\omega=2 \pi f$ and $\phi$ is the eigenvector. conditions. The other information from the old file of straight pipe is written into the new '*.inp' file by Matlab as well.

The automatic procedure for calculating the resonant frequencies of $2 \mathrm{D}$ networks is: create the '*.inp' by Matlab, submit it to Abaqus by commands in 225 Matlab, and then post-process the Abaqus output script in Matlab. This Mat- 
lab programme is named as '2DNRF' (for 2D Network Resonance Frequency), which will be used in section 4 .

\section{Optimization of network structures}

The value and number of resonant frequencies vary according to the netpositions depend on the end positions. If the end positions are known, the junction positions are known. The 2D network considered in this work consists of the three types of junctions shown in Fig $6 \mathrm{a} 6 \mathrm{c}$.

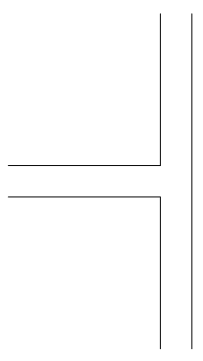

(a)

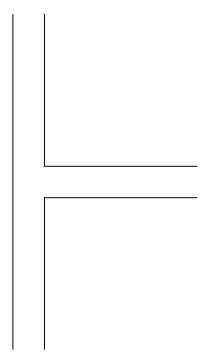

(b)

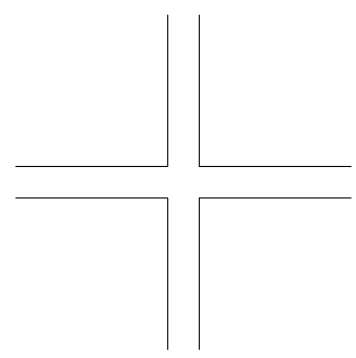

(c)

Figure 6: (a) T junction with left branch; (b) T junction with right branch; (c) Cross junction

Different networks can be formed by using different parameters. Their resonant frequencies can be estimated by the finite element method 2DNRF proposed before. By changing the parameters, networks with the wanted resonant frequencies or a maximum number of resonant frequencies can be found. Optimization methods should be adopted to optimize the parameters. Genetic algorithm (GA) is used for this purpose in this article but other optimization methods such as Simulated Annealing could also be used. 
The chromosome representations should be decided first. GAs operate on a number of potential solutions, called a population of chromosomes, consisting of some encoding of the network parameter set. The binary and integer chromosome representations are used. For a network each parameter of junction type is encoded as an integer in 0,1 or 2 which represent the junction types in Fig 6 a 6c, and the other parameters of network dimensions (such as end positions, cross-sections and so on) are encoded as binary strings.

Then the initial population can be generated by using a random number generator. There are two parts in a population, a binary part of network dimensions and an integer part of junction types. We can create a random binary matrix of size Nind $\times$ Lind for the parameters of network dimensions, where Nind specifies the number of individuals and Lind the length of the individuals. For the parameters of junction types, we can give an integer $(0,1$ and 2$)$ matrix of size Nind $\times$ Lind $^{j u n}$ where Lind ${ }^{j u n}$ is the number of junctions. By combining these two matrices, the initial population is obtained.

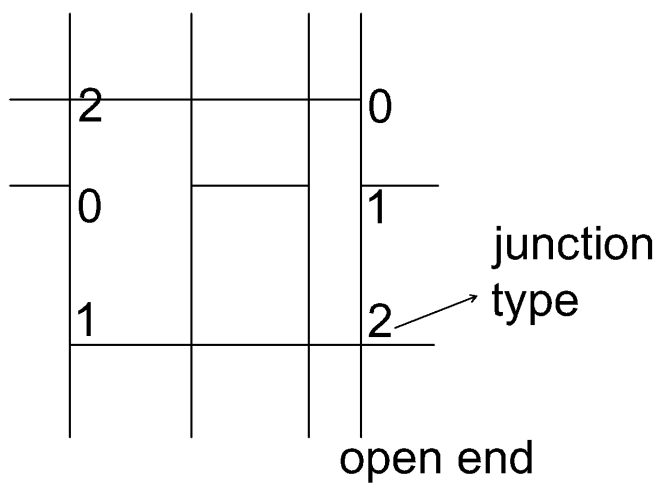

Figure 7: An example of network for the chromosome representation

Here is an example of an individual in a population of networks

$$
[\overbrace{0100 \ldots 1111, \underbrace{0101 \ldots 0101}_{l}, \ldots \ldots, 0101 \ldots . .0111}^{m}, \overbrace{2,1,0, \ldots \ldots, 1,0,2}^{n}] \text { network } i
$$

This is the chromosome representation of the network in Fig.7 The network has 3 rows and 4 columns. In the first part of the chromosome, there are $m$ 
dimension parameters and each one has $l$ binary strings which can be converted to a real value. So there are $m$ real values, and for this network $m$ is 8 . We specify the boundary of the network, so the open ends move only on this boundary and along with the junctions. Therefore, we only need to generate the real values for the coordinates of the junctions and the cross sectional area of the network. Since we use the same cross section for the network, only one real value is needed for the area. The other seven real values represent the positions of the three rows and four columns which determine the coordinates of the junctions. In the second part of the individual there are $n=12$ junctions and each integer represents the junction type. If this population consists of $k$ networks, its chromosome representation is a $k \times(m+n)$ matrix.

The purpose of this work is to find the wanted resonant frequencies or as many resonant frequencies as possible by designing and optimizing the network. The 2D FEM, implemented in the MATLAB code 2DNRF proposed before, has been used for estimating values of $f_{r}$ and the number of resonant frequencies of a network. If $f_{w}$ is the wanted resonant frequency and there are several resonant frequencies $f_{r 1}, f_{r 2}, \ldots$ for the network $i$, the objective function for the first purpose is given by

$$
\operatorname{Obj} V_{1}\left(x_{i}\right)=\min \left(\left|f_{r 1}-f_{w}\right|,\left|f_{r 2}-f_{w}\right|, \ldots\right)
$$

where $x_{i}$ is the chromosome of network $i$.

If the purpose is to find as many resonant frequencies as possible, the objective function is the number of resonant frequencies. A minus sign is added to get a minimization problem. One has

$$
\operatorname{Obj}_{2}\left(x_{i}\right)=-\operatorname{count}\left(f_{r 1}, f_{r 2}, \ldots\right)
$$
rameters of the network (dimensions and junction types).

For the minimization problem, the most fit individuals have the lowest numerical values of the associated objective function. The raw objective values in equation (8) or (9) are usually only used as an intermediate stage in determining 
transform the objective function value into a measure of relative fitness. Here, individuals are assigned a fitness according to the rank of their raw objective values in the population rather than the raw performance.

A population is first initialized and then improved through repetitive application of the mutation, crossover and selection operators. This process is repeated until a fixed number of generations has been reached. If we are not satisfied with the results, we can adjust the specified parameters of GA or networks and then run the optimization program again until we get targeted resonant frequencies.

\section{Design examples}

In order to illustrate that GA works for the optimization of $2 \mathrm{D}$ networks and that the resonant frequencies can be obtained by the GA process, some examples are given.

All the dimension parameters of the network can be optimized within given ranges during the GA process, but for the sake of simplicity some of them are specified. The network to be optimized in this section has 14 open ends and 12 junctions. The junction positions are arranged in four columns and three rows, and their coordinates depend on end coordinates. In Fig 8 there is an example of networks generated randomly in the first generation of the GA procedure. In Fig 8 we can see 14 positions for the open ends, but the network has 12 open ends due to the junction types. The circles represent the junction positions. Three types of junctions introduced before can be seen in this example. They are $\mathrm{T}$ junction with left branch, $\mathrm{T}$ junction with right branch and cross junction.

The corrections of open ends can be obtained by the methods introduced before. If the ends are closed, there is no need to add end corrections to the 305 original pipe lengths.

\subsection{Unflanged networks}

In this example there is no flange, so the end corrections, $0.001684 m$, can be calculated by equation (10) which is from paper [27.

$$
\delta_{s q 0}=0.597 a_{e f f}
$$




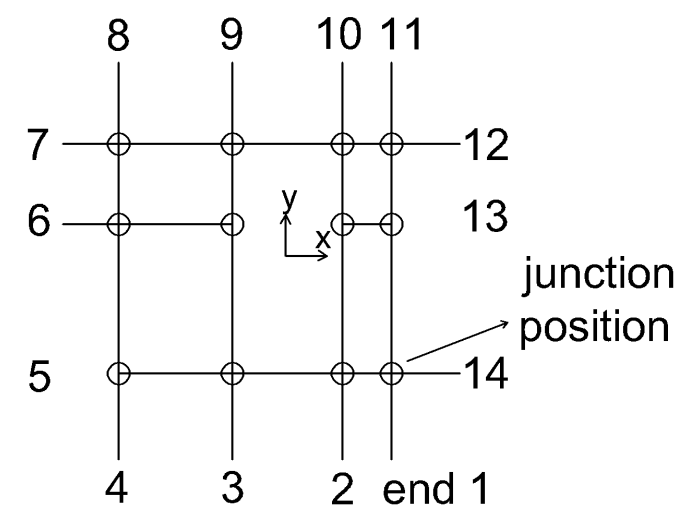

Figure 8: An example of networks generated randomly in the first generation of the GA procedure

with $a_{e f f}=2 a_{s q} / \sqrt{\pi}$ where $2 a_{s q}$ is the pipe width.

Transverse ( $\mathrm{x}$ axis) and some of longitudinal coordinates of the ends are given in table 1 . The other longitudinal coordinates of the ends vary within the ranges which are shown in table 1 as well. The pipe cross-sections are the same, $0.005 m \times 0.005 m$. Boundary conditions $p=0$ should be applied to the open ends, where $p$ is the acoustic pressure.

Table 1: Coordinates of central points of open ends of a unflanged network

\begin{tabular}{cccccc}
\hline End & $\mathbf{x}(\mathbf{m})$ & $\mathbf{y}(\mathbf{m})$ & End & $\mathbf{x}(\mathbf{m})$ & $\mathbf{y}(\mathbf{m})$ \\
\hline 1 & 0.0475 & -0.051684 & 8 & -0.0475 & 0.051684 \\
2 & 0.0325 & -0.051684 & 9 & -0.0325 & 0.051684 \\
3 & -0.0325 & -0.051684 & 10 & 0.0325 & 0.051684 \\
4 & -0.0475 & -0.051684 & 11 & 0.0475 & 0.051684 \\
5 & -0.076684 & {$[-0.0475,-0.015]$} & 12 & 0.076684 & {$[0.015,0.0475]$} \\
6 & -0.076684 & {$[-0.01,0.01]$} & 13 & 0.076684 & {$[-0.01,0.01]$} \\
7 & -0.076684 & {$[0.015,0.0475]$} & 14 & 0.076684 & {$[-0.0475,-0.015]$} \\
\hline
\end{tabular}

By giving the GA parameters, such as the number of individuals, the generation gap, the maximum number of generations and the wanted resonant 
found. This network has the closest resonant frequency to the targeted resonant frequency. The GA parameters should be adjusted for each case to obtain acceptable results.

Table 2: Results for the targeted resonant frequencies

\begin{tabular}{cccc}
\hline Case & Targeted(Hz) & Obtained $(\mathbf{H z})$ & Error $(\%)$ \\
\hline 1 & 1250 & 1254 & 0.32 \\
2 & 1400 & 1400 & 0 \\
3 & 1600 & 1590 & 0.625 \\
\hline
\end{tabular}

In table 2, the wanted resonant frequencies are obtained by optimizing the network structure and the maximum error is less than 1\%. The network structures can be seen in Fig 9a $9 \mathrm{c}$. Besides the resonant frequencies shown in table 2 these three networks have other resonant frequencies within $2000 \mathrm{~Hz}$. They are $1926 \mathrm{~Hz}$ in case $1,1976 \mathrm{~Hz}$ in case 2 and $1947 \mathrm{~Hz}$ in case 3 .

In Fig, 10 we can see the minimum difference between the targeted frequency $1400 \mathrm{~Hz}$ and the resonant frequencies of individuals in each generation in GA procedure. In the first generation the difference is $259.52 \mathrm{~Hz}$. From the second generation it becomes $6.93 \mathrm{~Hz}$. In the 7 th generation a very good result is found. The difference between its resonant frequency and the targeted frequency $1400 \mathrm{~Hz}$ is $0.22 \mathrm{~Hz}$. The number of individuals in each generation used in the

330 GA procedure is 1000 . This leads to a computing time of about two hours to get the final result by the GA algorithm.

\subsection{Flanged networks}

In this example the network is between the cylinder and the plane surface used in section 2. The calculations of open end corrections are presented before.

335 The end corrections of longitudinal open ends can be calculated by formula (5). For the transverse ends their corrections, $0.0054 m, 0.0057 m$, and $0.0054 m$, are given by formula 3 . 


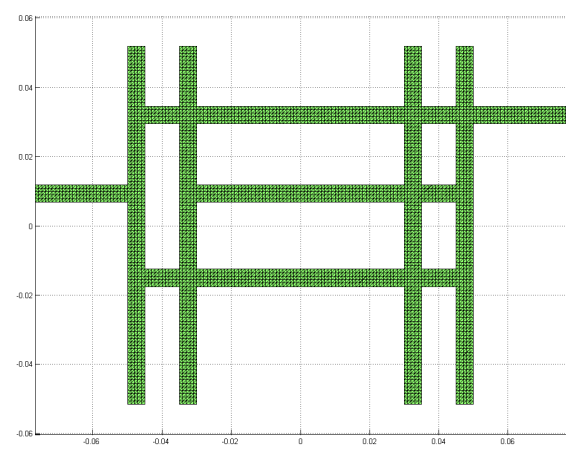

(a)

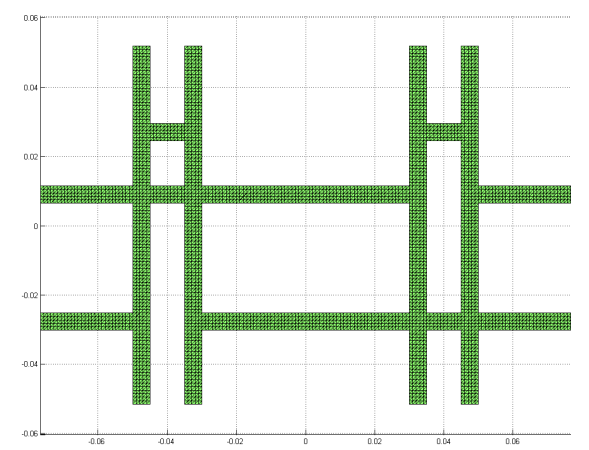

(b)

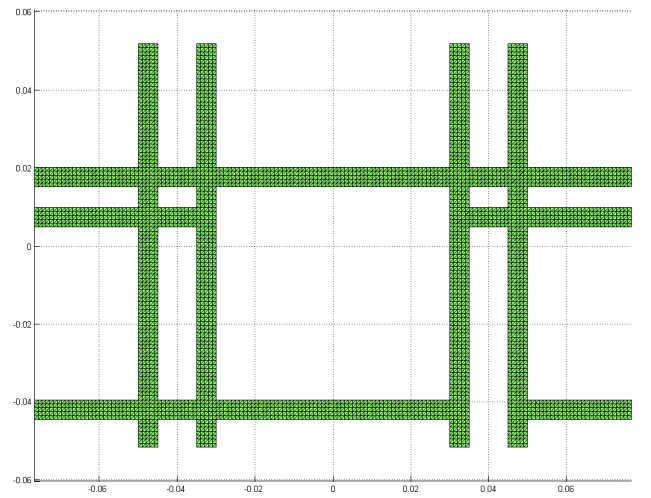

(c)

Figure 9: (a) Network with the resonant frequency $1254 \mathrm{~Hz}$; (b) Network with the resonant frequency $1400 \mathrm{~Hz}$; (c) Network with the resonant frequency $1590 \mathrm{~Hz}$.

Longitudinal and some transverse coordinates of the ends are given in table 3. The other transverse coordinates vary within the ranges which are shown in table 3. The pipe cross-sections are the same, $0.009 m \times 0.009 m$. Boundary conditions $p=0$ should be applied to the open ends, where $p$ is the acoustic pressure. In table 3, EC means the value of longitudinal end correction, which can be calculated by formula (5).

In this section we try to get networks with the maximum number of reso345 nant frequencies within the frequency range $0-2000 \mathrm{~Hz}$. With the dimension parameters of networks given before, networks with as many resonant frequen- 


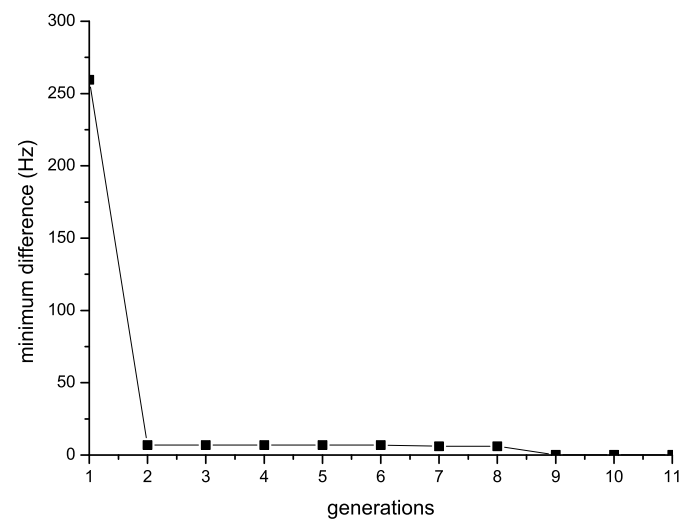

Figure 10: The minimum difference between the targeted frequency $1400 \mathrm{~Hz}$ and the resonant frequencies of individuals in each generation in the GA procedure

cies as possible will be searched by GA. The networks of interest have repeated junctions in the longitudinal direction ( $\mathrm{y}$ axis in Fig 8). It means that in each column of the network in Fig, 8 the junctions should be the same.

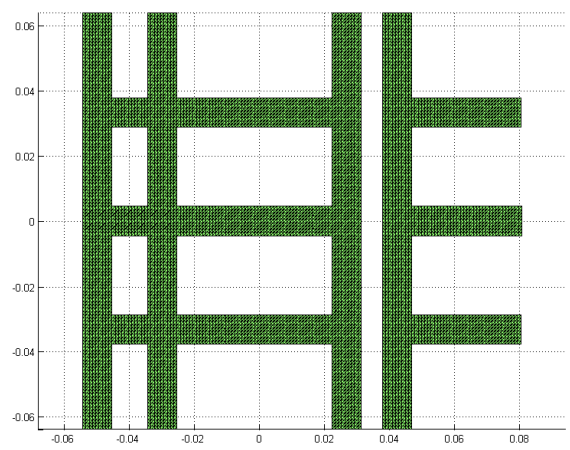

(a)

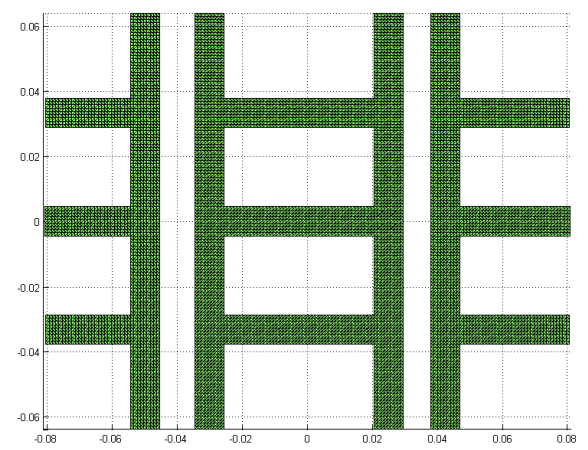

(b)

Figure 11: (a) Network 1; (b) Network 2.

By changing the GA parameters and running the GA program several times, four resonant frequencies at most within $0-2000 \mathrm{~Hz}$ are found. There are 2 networks with 4 resonant frequencies, and they are shown in Fig 11a and Fig $11 \mathrm{~b}$ The resonant frequencies are shown in table 4. It was found that different runs of the software lead to networks with quite similar structures but 
Table 3: Coordinates of central points of open ends of the network between a cylinder and a plane surface

\begin{tabular}{c|cc||c|cc}
\hline End & $\mathbf{x}(\mathbf{m})$ & $\mathbf{y}(\mathbf{m})$ & End & $\mathbf{x}(\mathbf{m})$ & $\mathbf{y}(\mathbf{m})$ \\
\hline 1 & {$[0.042,0.0705]$} & $-0.05-E C$ & 8 & {$[-0.0705,-0.042]$} & $0.05+E C$ \\
2 & {$[0.0045,0.033]$} & $-0.05-E C$ & 9 & {$[-0.033,-0.0045]$} & $0.05+E C$ \\
3 & {$[-0.033,-0.0045]$} & $-0.05-E C$ & 10 & {$[0.0045,0.033]$} & $0.05+E C$ \\
4 & {$[-0.0705,-0.042]$} & $-0.05-E C$ & 11 & {$[0.042,0.0705]$} & $0.05+E C$ \\
5 & -0.0804 & -0.03333 & 12 & 0.0804 & 0.03333 \\
6 & -0.0807 & 0 & 13 & 0.0807 & 0 \\
7 & -0.0804 & 0.03333 & 14 & 0.0804 & -0.03333 \\
\hline
\end{tabular}

Table 4: Networks with four resonant frequencies

\begin{tabular}{ccccc}
\hline Resonant frequency & 1st & 2nd & 3rd & 4th \\
\hline Network 1 & $1055 \mathrm{~Hz}$ & $1797 \mathrm{~Hz}$ & $1799 \mathrm{~Hz}$ & $1929 \mathrm{~Hz}$ \\
Network 2 & $1201 \mathrm{~Hz}$ & $1799 \mathrm{~Hz}$ & $1890 \mathrm{~Hz}$ & $1989 \mathrm{~Hz}$ \\
\hline
\end{tabular}

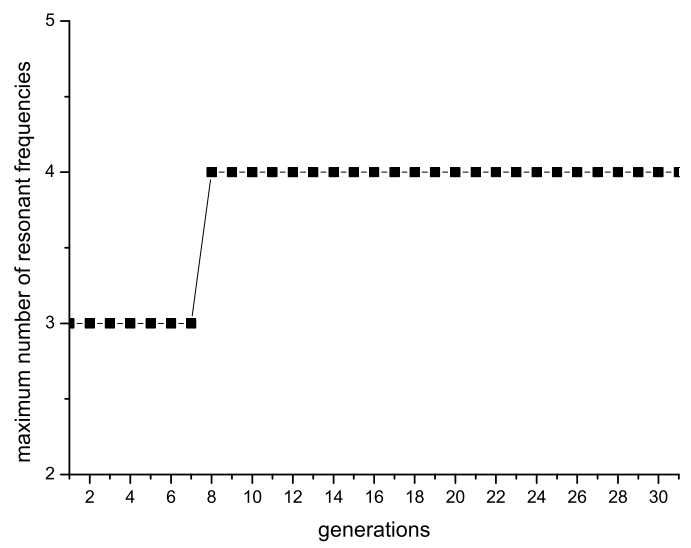

Figure 12: The maximum number of resonant frequencies for each generation in the GA procedure

with slightly different coordinates of the junctions.

In Fig 12, the maximum number of resonant frequencies of a network in the first generation is 3 . It changes to 4 in the $8 t h$ generation and then keeps 
the same value until the end of the GA procedure. Since we are interested in periodic networks, only networks with repeated junctions could be generated in each generation. So the number of individuals in each generation is just 30 .

From the above examples one can conclude that the GA process developed before is suitable for the optimization and design of networks. Once the ranges of dimension parameters of networks are given, networks with targeted resonant frequencies can be found. The design examples will be validated by measurements in section 6 ,

\section{Experimental validation}

Wooden networks are built and measured in order to validate the optimization in section 5. First a network without flange is measured. This network is optimized and built to target the resonant frequency $1400 \mathrm{~Hz}$. Then a network between a cylinder and a plane surface is built and measured to search 4 resonant frequencies predicted in section 4.

\subsection{Unflanged network}

An unflanged network in Fig 13a is measured in order to validate the optimization. The network in Fig $13 \mathrm{a}$ is built with wood. A speaker and a micro-

375 phone are put near the open ends in Fig 13a. The optimized network is a 2D network, so the depth is not given in the optimization. In the experiments, the depth that we use is $0.01 \mathrm{~m}$.

The SPL obtained by the microphone is shown in Fig 13b. We can see a deep valley at frequency $1370 \mathrm{~Hz}$ which is close to the targeted resonant frequency $1400 \mathrm{~Hz}$. So the GA optimization procedure developed before can give the resonant frequencies that we want.

\subsection{Flanged network}

The network between the cylinder and the plane surface is optimized in section 5.2. The acoustic fields of network 1 in Fig $11 \mathrm{a}$ are calculated and 


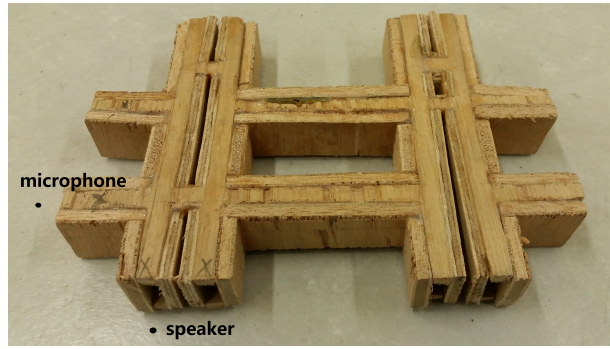

(a)

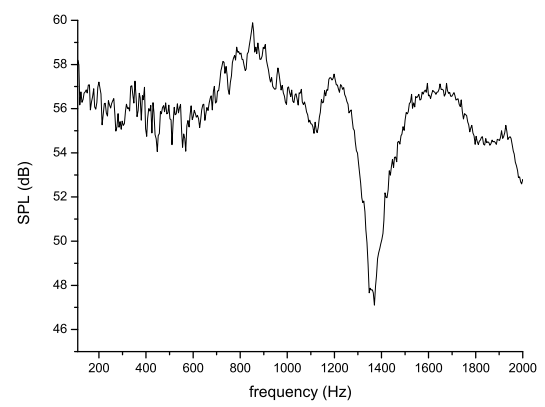

(b)

Figure 13: (a) Optimized wooden network with the resonant frequency $1400 \mathrm{~Hz}$; (b) Measured SPL for the optimized wooden network with the resonant frequency $1400 \mathrm{~Hz}$.

measured in order to validate the GA optimization methods. When we measure the case without network, we close the pipe ends in Fig $14 \mathrm{~b}$ with woods.

In our calculations, a dimensionless point source is used, and it has been assumed omni-directional. The general tendencies and frequency-dependant variations of the sound pressure will be compared between the predicted and measured results. Such comparisons are very common and widely used in previous studies such as in 29] and 14. So it is not necessary to use a omnidirectional source in our experiments. A numerical signal is generated and then sent to a real loudspeaker. This is the source used in these experiments. Its dimensions are $0.12 m \times 0.12 m \times 0.12 m$.

The radius and width of the cylinder are $0.27 \mathrm{~m}$ and $0.15 \mathrm{~m}$. Its location can be seen in the sketch of the experimental setup Fig.14a Sweep signals within $2000 \mathrm{~Hz}$ are generated by a generator. The center of the speaker is at $(1 \mathrm{~m}, 0 \mathrm{~m}, 0.06 \mathrm{~m})$. The microphone is at $(0.1 \mathrm{~m}, 0 \mathrm{~m}, 0.005 \mathrm{~m})$.

The predicted results are shown in Fig $15 \mathrm{a}$. The resonant frequencies of this optimized network are given in section 5.2 , which are $1055 \mathrm{~Hz}, 1797 \mathrm{~Hz}, 1799 \mathrm{~Hz}$ and $1929 \mathrm{~Hz}$. The second and the third resonant frequencies are very close, so just one extremum can be seen around $1800 \mathrm{~Hz}$ in Fig $15 \mathrm{a}$, where we can see two other extrema around $1055 \mathrm{~Hz}$ and $1929 \mathrm{~Hz}$. 


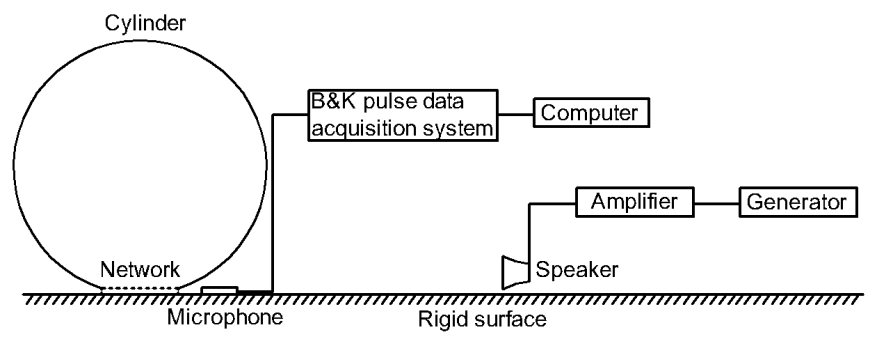

(a)

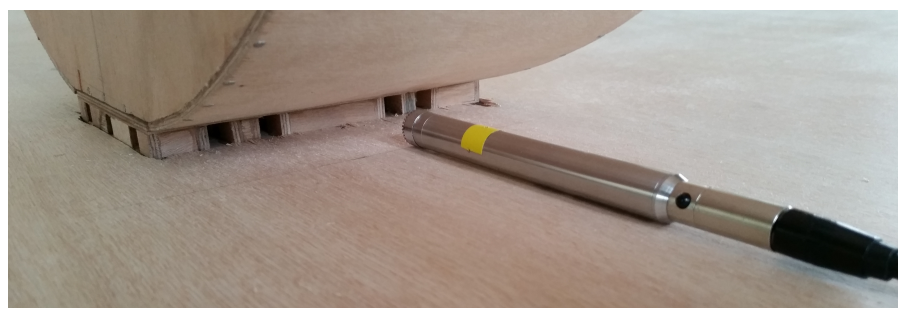

(b)

Figure 14: (a) Sketch of experimental setup; (b) An optimized network between a cylinder and a plane surface.

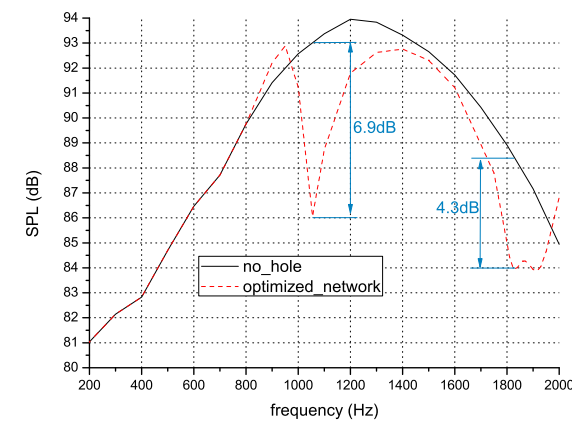

(a)

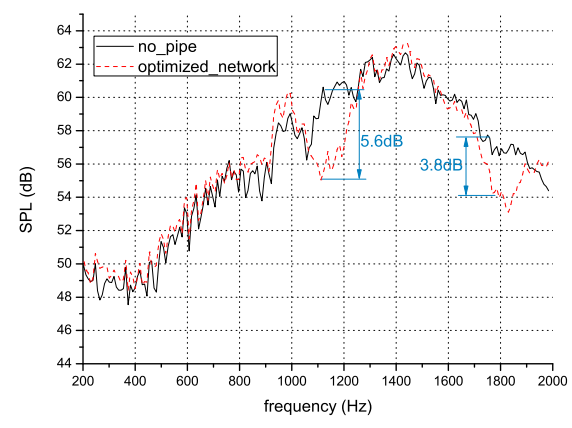

(b)

Figure 15: (a) Predicted results of an optimized network between a cylinder and a plane surface; (b) Measured results of an optimized network between a cylinder and a plane surface.

The measured results are given in Fig $15 \mathrm{~b}$, where the variations of SPL are similar to the predicted results in Fig 15 a. Around the wanted resonant frequencies, reductions of the measured SPL are close to reductions of the predicted 
SPL. The lower dip in the experimental curve compared to the computation can be explained by the damping in the system coming for instance from the wood material. From the predicted and measured SPL we can see that the optimized wooden network has the resonant frequencies that we want.

\section{Conclusions}

A numerical method for the calculation of resonant frequencies of network resonators has been proposed. Based on this numerical method, an optimization method using genetic algorithm is developed in order to get the targeted resonant frequencies of the network resonators. The optimization method is validated by experiments of design examples of wooden network resonators. The measured resonant frequencies of the optimized network resonators agree well with the targeted resonant frequencies. The good agreements also prove that the end corrections are estimated correctly.

Tire treads and road textures in the contact zones between tires and roads can be considered as networks. The network resonance is an important mechanism of the tire/road noise. With the methods proposed in this work, it is possible to investigate the influence of these networks on the tire/road noise and to design tire treads and road textures.

For a real tire the method could be applied by first estimating the end corrections of the pipe. This will need a fine mesh of the tire surface and potentially large computations but efficient methods such as BEM based on Fast Multipole Methods could help to get results in a reasonable time. Computations of resonant frequencies of the network in the contact zone should be done without too much difficulties. The method can also be applied to estimate the influence of the road surface of the acoustic radiation. This needs to describe the road asperities and to mesh a part of the road in the boundary element model. 


\section{Acknowledgment}

This work was funded by the China Scholarship Council (CSC) and by the

French Environment and Energy Management Agency (ADEME).

\section{References}

[1] U. Sandberg, J. A. Ejsmont, Tyre/road noise reference book, INFORMEX, 2002.

[2] C. Zwikker, C. Kosten, Sound Absorbing Materials: by C. Zwikker and C.W. Kosten, Elsevier Publishing Company, 1949.

[3] H. Tijdeman, On the propagation of sound waves in cylindrical tubes, Journal of Sound and Vibration 39 (1) (1975) 1-33.

[4] F. J. M. van der Eerden, Noise reduction with coupled prismatic tubes, Ph.D. thesis, University of Twente, Enschede, the Netherlands (November 2000).

[5] C. Field, F. Fricke, Theory and applications of quarter-wave resonators: A prelude to their use for attenuating noise entering buildings through ventilation openings, Applied Acoustics 53 (1) (1998) 117-132.

[6] F. Fahy, C. Schofield, A note on the interaction between a helmholtz resonator and an acoustic mode of an enclosure, Journal of Sound and Vibration 72 (3) (1980) 365-378.

[7] W. Slaton, J. Zeegers, Acoustic power measurements of a damped aeroacoustically driven resonatora), The Journal of the Acoustical Society of America 118 (1) (2005) 83-91.

455

[8] A. Cummings, The effects of a resonator array on the sound field in a cavity, Journal of Sound and Vibration 154 (1) (1992) 25-44.

[9] A. Doria, Control of acoustic vibrations of an enclosure by means of multiple resonators, Journal of Sound and Vibration 181 (4) (1995) 673-685. 
[10] O. Inácio, L. Henrique, J. Antunes, et al., Design of duct cross sectional areas in bass-trapping resonators for control rooms, Noise control engineering journal 55 (2) (2007) 172-182.

[11] P. Merkli, Acoustic resonance frequencies for a t-tube, Zeitschrift für angewandte Mathematik und Physik ZAMP 29 (3) (1978) 486-498.

[12] D. Li, J. S. Vipperman, On the design of long t-shaped acoustic resonators, the Journal of the Acoustical Society of America 116 (5) (2004) 2785-2792.

[13] D. Li, Vibroacoustic behavior and noise control studies ofadvanced composite structures, Ph.D. thesis, University of Pittsburgh (2003).

[14] G. Yu, Acoustic resonators for noise control in enclosures: modelling, design and optimization, Ph.D. thesis, The Hong Kong Polytechnic University (2009).

[15] D. Li, J. S. Vipperman, Noise control of mock-scale chambercore payload fairing using integrated acoustic resonators, Journal of spacecraft and rockets 43 (4) (2006) 877-882.

[16] S. A. Lane, R. E. Richard, S. J. Kennedy, Fairing noise control using tubeshaped resonators, Journal of spacecraft and rockets 42 (4) (2005) 640-646.

[17] R. E. Hayden, Roadside noise from the interaction of a rolling tire with the road surface, The Journal of the Acoustical Society of America 50 (1A) (1971) 113-113.

[18] C. Deffayet, et al., Air-pumping phenomena in road cavities, in: INTROC 90-International Tire/Road Noise Conference 1990, Gothenburg, Sweden, 1990.

[19] D. Ronneberger, Experimentelle und theoretische untersuchungen spezieller mechanismen der rollgeräusche, Mitteilungen des Instituts für Straßen-, Eisenbahn-und Felsbau an der ETH Zürich 57 (1984) 55. 
[20] D. RONNEBERGER, Towards quantitative prediction of tyre/road noise, in: workshop on rolling noise generation, Institut fur Technische Akustik, Technische Universitat Berlin., 1989.

[21] W. Kropp, F. Becot, S. Barrelet, On the sound radiation from tyres, Acta Acustica united with Acustica 86 (5) (2000) 769-779.

[22] R. Graf, C. Kuo, A. Dowling, W. Graham, Horn amplification at a tyre/road interface-part i: experiment and computation, in: Proceedings of internoise 99-the 1999 international congress on noise control engineering held in fort lauderdale, Florida, USA, 6-8 December 1999, volume 1, 1999.

[23] R. Graf, C. Kuo, A. Dowling, W. Graham, On the horn effect of a tyre/road interface, part i: Experiment and computation, Journal of Sound and Vibration 256 (3) (2002) 417-431.

[24] F. Anfosso-Ledee, Tyre / road noise horn effect : 2d modelling by analytical and numerical models, Tech. rep., national PREDIT project (2000).

[25] F. Anfosso-Ledee, P. Klein, A. Fadavi, D. Duhamel, Tire/road noise: comparison of $2 \mathrm{~d}$ and $3 \mathrm{~d}$ models for horn effect, in: Internoise 2000. Proceedings of the 29th international congress on noise control engineering, held 27-31 August 2000, Nice, France., Vol. 1, 2000.

[26] A. Fadavi, Tire/road noise: 3d model for horn effect, in: The 29th International Congress and Exhibition on Noise Control Engineering, 2000.

[27] J.-P. Dalmont, C. Nederveen, N. Joly, Radiation impedance of tubes with different flanges: numerical and experimental investigations, Journal of sound and vibration 244 (3) (2001) 505-534.

[28] D. Duhamel, L'acoustique des problèmes couplés fluide-structure - application au contrôle actif du son, Ph.D. thesis, Ecole Nationale des Ponts et Chaussées (1994). 
[29] A. Cummings, The effects of a resonator array on the sound field in a cavity, Journal of Sound and Vibration 154 (1) (1992) 25-44. 